\title{
¿La transparencia de las páginas web ayudan en la captación de clientes? Análisis de los campos de golf en Andalucía
}

\author{
Marta García-Tascón(D, Marcos Pradas-García (D) \\ Facultad Ciencias del Deporte. Departamento Deporte e Informática. Universidad Pablo de Olavide (Spain) \\ margata@,upo.es, marcos.pradas@.gmail.com
}

Received April, 2016

Accepted May, 2016

\section{Resumen}

Objeto: El objetivo de este estudio consiste en analizar el grado de transparencia de las páginas Web de las organizaciones que pertenecen al mundo del deporte, en concreto de los campos de golf de Andalucía.

Diseño/metodología/enfoque: Se ha realizado un análisis descriptivo de las condiciones de transparencia de las 92 páginas Web de los clubes de golf inscritos en la página Web oficial de la Real Federación de Golf de Andalucía (RFGA). Se utiliza un cuestionario creado/adaptado ad hoc en base al trabajo de Barrio y Martín (2012) que analizan el cumplimiento de las 4 condiciones de transparencia.

Aportaciones y resultados: Se reflejan un alto grado de transparencia, pues muestran un cumplimiento igual o superior al 75\% en 7 de las 11 variables. La página Web se convierte en un elemento para cuantificar el rendimiento del campo sin necesidad de invertir en exceso en otros canales de promoción para atraer al mayor número de posibles clientes.

Limitaciones: En algunas páginas Web el acceso a la información conllevaba una importante inversión de tiempo, pues no tenían la característica de ser intuitivas y de fácil "visibilidad del contenido". En algunos casos no se disponía de toda la información requerida. 
Implicaciones prácticas: El conocimiento de esta información permite al director o gerente del campo detectar las posibles áreas de mejora para así optimizar su gestión, a la vez que se mejora la transparencia ofrecida por los campos de golf.

Implicaciones sociales: Se pone de manifiesto la necesidad de enseñar al usuario a evaluar la calidad, transparencia y ser crítico con la información que encuentra en las páginas Web.

Originalidad / Valor añadido: El análisis de la transparencia permite a los clubes ser el reflejo de sus campos de golf. Se convierte en la herramienta que el gerente puede utilizar para mostrar parte de todas sus virtudes y para evaluar el comportamiento social de sus clientes, atraer a otros posibles y realizar acciones con los grupos de interés de la industria del golf.

Palabras clave: Transparencia, Internet, Páginas Webs, Golf, Andalucía

Códigos JEL: M39, R50

Title: Does the transparency of the websites can help in attracting customers? Analysis of the golf courses in Andalusia

\section{Abstract}

Purpose: The aim of this study is to analyze the degree of transparency of the Web pages of organizations that belong to the world of sports, specifically golf courses in Andalusia.

Design/methodology: We performed a descriptive analysis of the conditions of transparency of $92 \mathrm{Web}$ pages of golf clubs registered on the official website of the Royal Golf Federation of Andalusia (RFGA). A questionnaire created/adapted ad hoc is used for this study having as a reference the questionnaire used by Barrio and Martin (2012) that analyze the performance of the 4 conditions of transparency of Web pages.

Findings: To reflect a high degree of transparency, they show an equal to or higher compliance than $75 \%$ in 7 of the 11 variables. The Web page becomes an element to quantify the performance of the field without investing too much in other promotional channels to attract as many customers as possible. 
Research limitations/implications: In some Web pages, the access to information entailed a significant investment of time because they did not have the characteristic of being intuitive and easy "content visibility". In some cases was not available all required information.

Practical implications: Knowledge of this information allows the director or manager identifies possible areas of improvement to optimize their management, while transparency offered by the golf courses is improved.

Social implications: This study highlights the need for teaching the user to evaluate the quality, transparency and being critical with the information found on Web pages.

Originality/value: The analysis of transparency allows clubs to be reflective of its golf courses. It becomes the tool that the manager can use to display part of all its virtues and to evaluate the social behavior of their customers, to attract possible clients and perform actions with the stakeholders in the golf industry.

Keywords: Transparency, Internet, Websites, Golf, Andalusia

Jel Codes: M39, R50

\section{Introducción}

La repercusión del golf en el sector turístico es muy importante. España acoge aproximadamente 1,6 millones de turistas practicantes de golf cada año y llega a suponer el 10.6\% del PIB del país (Turespaña, Aymerich Golf Management e Internacional Golf Travel Market, 2005). Y en Andalucía se ha convertido en un negocio, que ya en el año 2011 supuso unos 680 millones de euros, cantidad superior al 17\% del producto interior bruto (ABC, 2011).

Así la página Web se ha convertido en un instrumento cada vez más importante para acceder y analizar la información de la organización (Borrero \& Cortijo, 2006). Para evaluar la calidad de la información en las páginas Web es necesario contar con unos criterios que permitan filtrar la información, y que sea fiable y contrastada. Además hay que tener en cuenta otros factores que pueden afectar a las páginas Web como son: los intereses comerciales, la falta de credibilidad, el fácil acceso y/o la escasa habilidad en la consulta por parte del usuario (Merlo-Vega, 2003). 
Hay ciertas investigaciones que analizan la calidad y cantidad de información que contienen las páginas Web empresariales a través del uso de los denominados índices de divulgación (Gandía, 2001; Giner, 1997; Larrán \& Giner, 2002), e indican de la necesidad de las empresas de comunicar sus resultados mediante internet para no quedar obsoletas y ser más accesibles (Gandía \& Andrés, 2005; Lymer, Debrency, Gray \& Rahman, 1999; Muñoz, Del Campo \& De La Fuente, 2001; Páez \& Oviedo, 2002; Rodríguez \& Sáez, 2005; Sánchez, 1999; Sierra \& Escobar, 1998).

Así, en algunas empresas del IBEX 35, los accionistas o el consejo de administración pueden solicitar una auditoria para que las cuentas tengan la mayor transparencia posible (Borrero \& Cortijo, 2006; Gandía, 2001; Larrán \& Giner, 2002). Otros estudios tratan de determinar las razones que llevan a las compañías a tomar la decisión de utilizar la página Web como instrumento de comunicación con los usuarios potenciales (Andrés \& Gandía, 2000; Asociación Española de Contabilidad y Administración de Empresas, 1988, 2002, 2004; Bonsón \& Escobar, 2004, 2006; Bonsón, Escobar \& Flores, 2006; Davis, Clements \& Kehuer, 2004).

Siguiendo a Sanz y Bernal (2008) pusieron de manifiesto que un nivel medio general sobre transparencia en página web, destacaban como sectores más transparentes el de servicios financieros y el de bienes de consumo, siendo el sector de materiales básicos, industria y construcción el que más asimetrías informativas presentaba. Por el contrario, no se pudo demostrar la relación entre transparencia y el nivel de capitalización bursátil de las empresas en el índice bursátil.

Sin duda, disponer de una página Web accesible y transparente es imprescindible para la promoción de los campos de golf (Larrán \& Giner, 2002). En estas organizaciones, los socios del club pueden solicitar que se realice una auditoría económica, pero la información es de régimen interno, y por tanto no tiene porqué ser publicada en la página Web, como indica la ley de la formación de clubes (Ley 10/1990, de 15 de octubre, del Deporte).

La Confederación de Empresarios de Andalucía (CEA) ha publicado diversos estudios donde relaciona el papel del golf dentro del turismo, pero nunca ha tenido en cuenta las páginas Webs como herramientas para su promoción (CEA, 2000).

Para medir la transparencia, el estudio realizado por FASB (Financial Accounting Standards Board) recoge en el documento Business Reporting Information (FASB, 2000) algunas razones por las que las empresas podrían estar interesadas en divulgar su información financiera a través de Internet, destacan: 
- Reducción de costes y tiempo en la divulgación de información a los sujetos interesados, lo que incrementa el valor de la información contable.

- Complementar las prácticas tradicionales de divulgación informativa.

- Incrementar y mejorar la cantidad y tipología de la información distribuida.

- Mejorar el acceso de los inversores potenciales a las pequeñas compañías.

Otra característica que Jadad y Gagliardi (1998) señalan, es que para que las páginas Web ofrezcan una información fiable deben cumplir los criterios de por ejemplo, que la información esté estructurada, clasificada y revisada.

Barrio y Martín (2012) definen que la transparencia se entiende como el esfuerzo por publicar y diseminar la información relevante de la organización, haciéndola visible y accesible a los diferentes grupos de interés de forma comprensible y actualizada. Por tanto se deben cumplir estas cuatro condiciones.

- Visibilidad del contenido: facilitar que el contenido sea captado de manera sencilla por estar situado en un lugar visible en las páginas webs.

- Accesibilidad: permitir que todas las personas, con independencia de su capacidad física o intelectual, puedan percibir, entender, navegar e interactuar con la Web, el contenido puede ser visible, pero si se necesita un permiso o registro para poder consultarlo no se puede considerar que la información sea accesible.

- Actualidad: si los contenidos no están al día, se estima que no hay una disposición real de ser transparente.

- Comprensibilidad: significa que la información esté escrita, al menos, en castellano.

Un sistema para establecer esta transparencia es el uso de los rankings, en el que se evalúa unos indicadores para identificar y clasificar a las instituciones o entidades de un sector más idóneas y adecuadas. Por ejemplo, destaca el ranking de las universidades donde por un lado informa a los posibles o futuros estudiantes y por otro orienta a los empresarios en la búsqueda de graduados o estudiantes de postgrado existentes (Martí, Puertas \& Calafat, 2014). 
También, de reciente actualidad es el análisis sobre la transparencia en diferentes tipos de organizaciones como, en las universidades españolas (Barrio \& Martin, 2012), en las fundaciones (Aguirre, 2013; Rodríguez \& Martín, 2013), en las páginas de los gobiernos (Eschenfelder, 2004; Heald, 2006; Meijer, 2009), en ONGs (Gálvez, Caba \& López, 2009), en los partidos políticos (Barrio, MartínCavanna y Martisi, 2013), que además viene impulsado con el cumplimiento a nivel nacional de la Ley 19/2013, de 9 de diciembre, Ley de Transparencia, Acceso a la Información Pública y Buen Gobierno.

Así como otros elementos de control como son: Orden Eco/3722/2003, sobre el informe anual de Gobierno Corporativo y otros instrumentos de información de las sociedades anónimas cotizadas y otras entidades de 2004 y/o el Proyecto de Ley de Transparencia Pública de Andalucía (2014).

Disponer de una página Web accesible y transparente es imprescindible para la promoción de los campos de golf y que su oferta llegue a los posibles clientes potenciales (turistas, touroperadores).

El objetivo de este estudio consiste en analizar el grado de transparencia de las páginas Web de los campos de golf de Andalucía.

\section{Metodología}

Se ha realizado un análisis descriptivo de las condiciones de transparencia de las páginas Web de los campos de golf de Andalucía.

Se ha creado/adaptado un cuestionario ad hoc (checklist) para el objeto de estudio, donde la respuesta es dicotómica (SI / NO) sobre el cumplimiento de las diferentes variables de análisis. Teniendo como referencia el cuestionario empleado por la Fundación Compromiso y Transparencia en el estudio realizado a las universidades españolas (Barrio \& Martín, 2012) que consta de 11 variables que analizan el cumplimiento de las 4 condiciones de transparencia de las páginas Web.

Se han desarrollado diferentes fases en la creación del cuestionario:

- FASE 1: Diseño del instrumento

Consulta de diferentes estudios realizados para la medición de la transparencia en las páginas webs (Aramayo-García, Arimany-Serrat, Uribe-Salaza \& Sabata-Aliberch, 2016; Bonsón, Escobar \& Gandía, 2004; Codina, 2000a, 2000b; Sánchez, 1999; Sierra \& Moreno, 2005). 
Elección del instrumento de Barrio y Martin (2012) y adaptación para realizar el estudio de análisis de las páginas Web de los campos de golf de Andalucía.

Para conocer el grado de cumplimiento de transparencia de la página Web, éstas deben cumplir con las 4 condiciones de: Visibilidad, Accesibilidad, Actualidad y Compresibilidad. Que se evalúan a través del cumplimiento de los criterios y 11 variables que las componen.

En la Tabla 1 se exponen los criterios que recoge el estudio en las universidades españolas (parte izquierda) y en la parte de la derecha se muestran los criterios seleccionados y adaptados (números 4 a 9) para los campos de golf (se los cambios).

Cada criterio está compuesto por diferentes variables que se repiten de forma habitual en las páginas webs de los campos de golf de Andalucía (Tabla 2).

\begin{tabular}{|l|l|}
\hline Criterios Universitarios & Criterios Campos de Golf \\
\hline 1. Misión & 1. Misión \\
\hline 2. Plan estratégico & 2. Plan estratégico \\
\hline 3. Personal & 3. Personal \\
\hline 4. Gobierno & 4. Organos de gobierno \\
\hline 5. Oferta y demanda académica & 5. Oferta y demanda \\
\hline 6. Claustro & 6. Información relevante para el socio \\
\hline 7. Alumnos & 7. Grado de satisfacción de usuarios \\
\hline 8. Resultados & 8. Resultados de los campos de golf \\
\hline 9. Información económica & 9. Rendición de cuentas \\
\hline
\end{tabular}

Tabla 1. Diseño instrumento con los criterios para el estudio de transparencia de universidades y campos de golf 


\begin{tabular}{|c|c|c|}
\hline $\begin{array}{l}\text { Condiciones transparencia } \\
\text { páginas Web }\end{array}$ & Criterios & $\begin{array}{l}\text { Variables que evalúan } \\
\text { la transparencia }\end{array}$ \\
\hline \multirow[t]{4}{*}{ Visibilidad } & $\begin{array}{l}\text { 1. Misión. La organizable trata de captar el mayor número de } \\
\text { socios para ser rentable y ofrecer así la mejor calidad }\end{array}$ & $\begin{array}{l}\text { 1. Acceso socios } \\
\text { 2. Registro socios }\end{array}$ \\
\hline & $\begin{array}{l}\text { 2. Plan estratégico. Refleja la forma de conseguir lograr el } \\
\text { objetivo de hacer el campo rentable }\end{array}$ & 3. Reserva online \\
\hline & $\begin{array}{l}\text { 3. Personal. Se exponen los servicios que ofrece el club y las } \\
\text { personas que trabajan en él }\end{array}$ & $\begin{array}{l}\text { 4. Servicios (escuela de } \\
\text { golf) }\end{array}$ \\
\hline & $\begin{array}{l}\text { 9. Rendición de cuentas. En las organizaciones públicas se } \\
\text { deben mostrar las cuentas anuales. En los campos privados solo } \\
\text { deben mostrar éstas de manera anual a su consejo de } \\
\text { administración }\end{array}$ & $\begin{array}{l}\text { 5. Tarifas (precios) y } \\
\text { ofertas }\end{array}$ \\
\hline Accesibilidad & $\begin{array}{l}\text { 4. Órganos de gobierno. Exponen las personas que dirigen en el } \\
\text { club y las instalaciones con las que cuenta }\end{array}$ & $\begin{array}{l}\text { 6. Instalaciones-Casa } \\
\text { Club }\end{array}$ \\
\hline \multirow[t]{3}{*}{ Actualización } & $\begin{array}{l}\text { 5. Oferta y demanda. Se informa de todas aquellas actividades } \\
\text { que ofrecen. Se debe indicar la demanda anual, número de } \\
\text { salidas vendidas, número usuarios de la escuela, etc... }\end{array}$ & 7. Calendario y eventos \\
\hline & $\begin{array}{l}\text { 7. Grado de satisfacción de usuarios. Recoge como expresan su } \\
\text { opinión los usuarios si les gusta el campo. Deben utilizarse } \\
\text { instrumentos que recogen la opinión del cliente/socio }\end{array}$ & 8. Contacto \\
\hline & $\begin{array}{l}\text { 8. Resultados de los campos de golf. Muestra como es el } \\
\text { recorrido del campo de golf, forma, diseño, etc... }\end{array}$ & $\begin{array}{l}\text { 9. Vista del campo, } \\
\text { fotos }\end{array}$ \\
\hline Comprensibilidad & $\begin{array}{l}\text { 6. Información relevante para el socio. Se refiere a aquellos } \\
\text { detalles que necesita el cliente/usuario para poder jugar de la } \\
\text { mejor manera posible }\end{array}$ & $\begin{array}{l}\text { 10. Meteorología } \\
\text { 11. Idioma }\end{array}$ \\
\hline
\end{tabular}

Tabla 2. Condición de transparencia, criterio de evaluación y variables de análisis de las páginas webs de los campos de golf de Andalucía

- FASE 2: Validación del instrumento

Para la validación de contenido, se remite por email la información de la Tabla 1 y Tabla 2 a un Grupo de expertos (10 personas) en materia deportiva y en el golf compuesto por 4 gerentes de campos de golf, 2 jugadores profesionales de golf, 2 greenkeepers y 2 profesores de universidad especialistas en gestión deportiva.

Los expertos remiten algunas sugerencias referidas a cambios de algunas palabras para facilitar la mejor comprensión del contenido.

\section{- FASE 3: Prueba piloto e Instrumento definitivo}

Se accede a la página Web de la Real Federación Española de Golf y en el apartado de clubes se seleccionan al azar 10 campos de golf para pasar el cuestionario y examinar si se podían analizar los diferentes apartados del cuestionario creado. Se realizó de forma satisfactoria este análisis. 


\subsection{Muestra de estudio}

Se ha realizado el análisis del total del universo de estudio que componen las 92 páginas Web de los clubes de golf inscritos en la página Web oficial de la Real Federación de Golf de Andalucía (RFGA). Se presenta un análisis de los campos de golf en función de la distribución por provincias y también por número de hoyos por provincia.

- Análisis de los campos en función de la distribución de las provincias (Figura 1) de las páginas Web analizadas

La provincia que cuenta con más campos de golf es Málaga (30.43\%) y la que menos es Jaén $(1.09 \%)$.

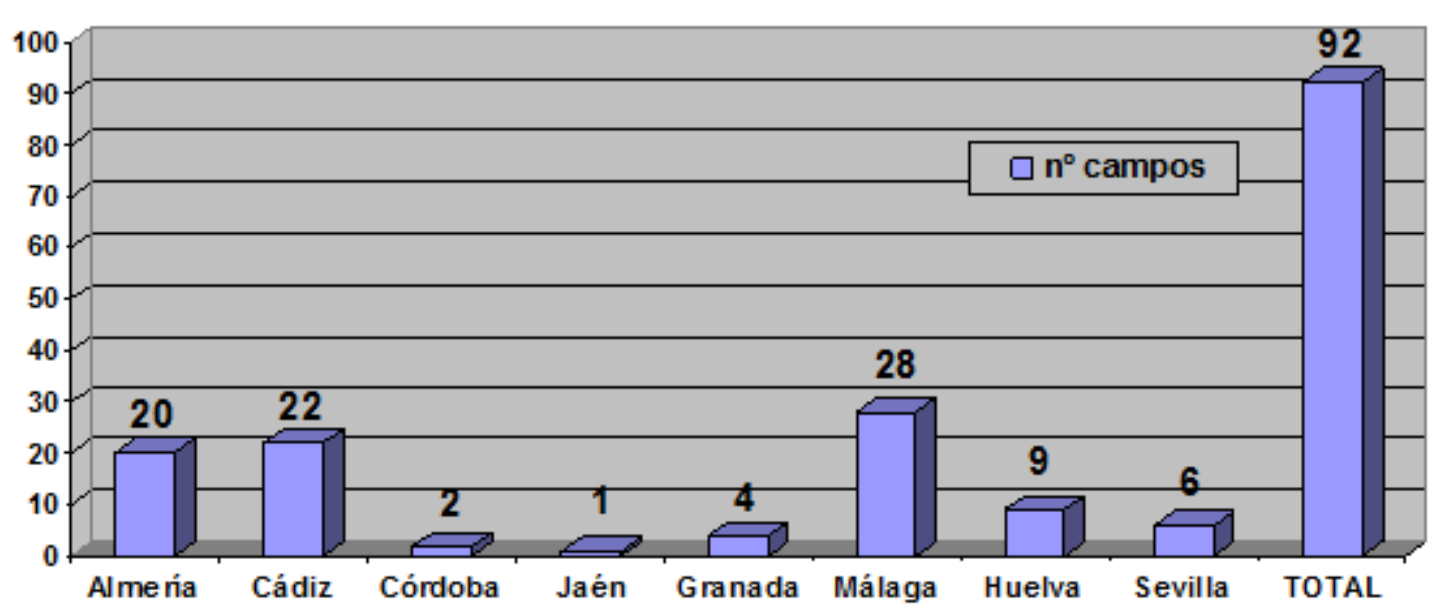

Figura 1. Campos de golf hoyos en Andalucía en función de la provincia

- Análisis de los campos en función del número de hoyos por provincia (Figura 2) de las páginas Web analizadas

Andalucía cuenta con 59 campos de golf con 18 hoyos (64.13\%) y 17 ofrecen juego en campos de 9 hoyos (18.48\%).También cuenta con un campo de 60 hoyos, y otro de 54 hoyos representando el $1.09 \%$ cada uno. Además de 6 campos de 27 hoyos $(6.52 \%)$ y 8 campos de 36 hoyos $(8.70 \%)$. 
Respecto a la distribución de estos campos en función de la provincia encontramos que la provincia de Málaga es la que más campos de 18 hoyos tiene y la que menos es Jaén. En cuanto a los campos de 9 hoyos, destaca la provincia de Málaga, y en el caso de la provincia de Granada y Almería, estos no cuentan con ninguno de estas características.

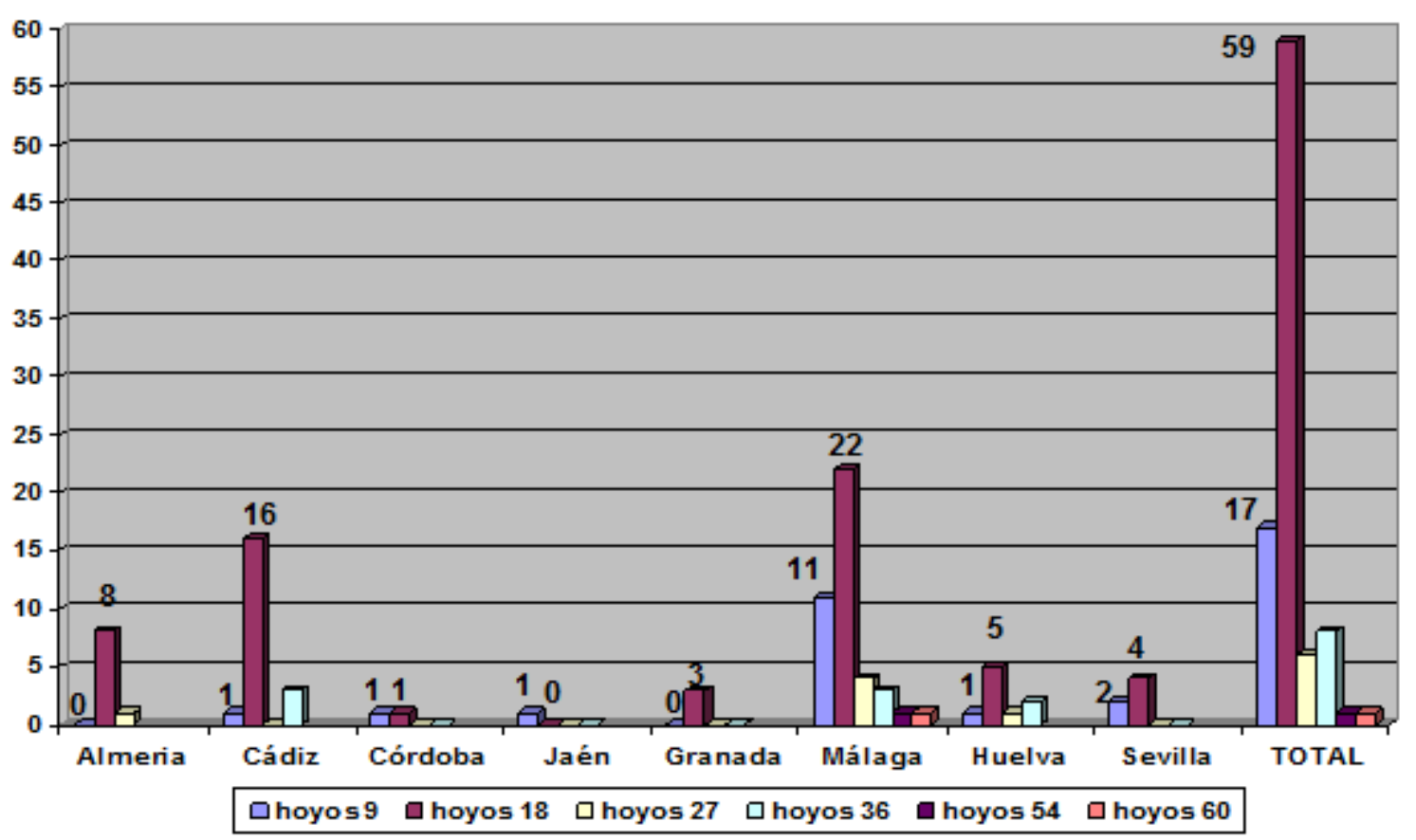

Figura 2. Campos de golf de 60, 54, 36, 27, 18 y 9 hoyos en Andalucía en función de la provincia

\subsection{Procedimiento y Análisis de datos}

El procedimiento seguido ha sido la revisión y análisis de las 92 páginas Web de los clubes de golf inscritos en la página Web oficial de la Real Federación de Golf de Andalucía (RFGA) durante el período de Enero a Junio de 2013, una segunda revisión durante los meses de Junio a Septiembre de 2014 y una tercera, hasta febrero de 2016 (en esta última no ha habido ningún tipo de variación).

Se analizan los datos mediante el sistema Microsoft Excel 2007 (v12.0) para Windows. 


\section{Resultados y Discusión}

En este apartado se muestran los resultados y discusión obtenidos a partir del análisis de transparencia de las 92 páginas Web de los campos de golf de Andalucía.

En la Tabla 3 se recoge la información más detallada de los resultados obtenidos en las variables analizadas.

\begin{tabular}{|c|c|}
\hline $\begin{array}{l}\text { Variables que evalúan la } \\
\text { transparencia }\end{array}$ & Porcentaje de cumplimiento \\
\hline $\begin{array}{l}\text { 1. Acceso } \\
\text { y } \\
\text { 2. registro a socios }\end{array}$ & $\begin{array}{l}\text { Existen } 77(83.70 \%) \text { páginas Web que facilitan el acceso a los socios para } \\
\text { realizar la reserva de campo, y en el } 52.17 \%(48) \text { de ellas, exigen al socio que esté } \\
\text { registrado para hacerlo }\end{array}$ \\
\hline 3. Instalaciones-Casa Club & Hay 80 páginas Web $(87 \%)$ que disponen de ellas y lo muestran \\
\hline 4. Vista del campo & Se ofrece en $55(59.8 \%)$ de las páginas Webs de los campos analizados \\
\hline 5. Servicios (escuela de golf) & El 95,6\% (88) ofrece este servicio \\
\hline 6. Reserva online & Se realiza en 61 de las páginas Web $(66.3 \%)$ \\
\hline 7. Tarifas (precios) y ofertas & En $77(80.4 \%)$ de las páginas Web de los campos muestran sus tarifas y ofertas \\
\hline 8. Contacto & $\begin{array}{l}\text { El } 92.4 \% \text { (85) indica al menos una forma de contacto, destaca el email y el } \\
\text { cuestionario de acceso }\end{array}$ \\
\hline 9. Calendario y eventos & En 65 páginas Web $(70.65 \%)$ se muestran \\
\hline 10. Meteorología & $\begin{array}{l}\text { Es recogida en el } 75 \% \text { (69) de las páginas Web, y refleja un periodo mínimo de } \\
72 \text { horas }\end{array}$ \\
\hline 11. Idioma & $\begin{array}{l}\text { En el } 100 \% \text { de las páginas Webs de los } 92 \text { campos ofrecen un idioma diferente } \\
\text { además del castellano, y en } 64 \text { campos }(69.5 \%) \text { frecen más de dos idiomas }\end{array}$ \\
\hline
\end{tabular}

Tabla 3. Análisis transparencia páginas Webs de los campos de golf de Andalucía

El análisis nos indica que los campos de golf de Andalucía han alcanzado una media del 66\% de cumplimiento de las diferentes condiciones sobre la transparencia las páginas Web, destacando Accesibilidad (87\%) y Comprensibilidad (81.5\%).

Se observa que en nueve de las variables analizadas, este cumplimiento es superior al $60 \%$, y en cinco superior al $80 \%$, destacando la variable del idioma con un cumplimiento del $100 \%$ al ofrecer los campos un idioma diferente al castellano en la página Web.

Los campos buscan mostrar al máximo todas sus virtudes para hacerse atractivos para el cliente mostrando datos sobre la información meteorológica en las próximas 72 horas y facilidades de contacto con el propio campo. 
Un aspecto de mejora es ofrecer la visión de los campos, hoyo a hoyo de manera virtual, ya que no llega al 60\%, los campos que lo muestran y es algo demandado por el usuario (Turespaña, Aymerich Golf Management e International Golf Travel Market, 2005).

Hay un porcentaje aún amplio para que los clubes cubran las necesidades de los usuarios de golf, por lo que el club debe trabajar con su página web, convertirla en un elemento transparente, atractivo y accesible, donde los usuarios sientan que al entrar en la página Web pueden encontrar toda la información relevante del campo de golf (Larrán \& Giner, 2002).

La bibliografía sobre todo refleja información sobre esponsorización de torneos de golf (Clark, Cornwell \& Pruitt, 2009; Berger et al., 2005). Para avanzar en la transparencia, el club debe poner a disposición pública la información económica y que, no sólo sea de régimen interno del club, al igual que lo exponen las empresas del Ibex 35 puesto que ningún club ha expuesto información sobre su actividad financiera, o el volumen de servicios que puedan llegar a ofrecer durante un año, o estrategias sobre como es la captación de clientes, etc.

\section{Conclusiones}

Se ha comprobado que la calidad de la información en las páginas Web de los campos de golf es alta, ya que cumple un alto porcentaje de criterios. Para evitar los problemas derivados de la calidad de la información y de la transparencia de las páginas Webs, se debería enseñar al usuario a evaluar la calidad de la información en internet a través de campañas u otros medios realizados por profesionales de la gestión deportiva.

Todas las páginas Web de los campos de golf andaluces se han analizado siguiendo las directrices del cuestionario del estudio de Barrio y Martín (2012). Como se ha observado, se refleja un alto grado de transparencia, pues muestran un cumplimiento igual o superior al $75 \%$ en 7 de las 11 variables.

Los campos deben trabajar en la mejora del acceso de los socios, para que no suponga una molestia para el usuario. El hecho de tener que registrarse como usuario en una página Web del club y facilitar datos personales, puede ocasionar la perdida de usuarios. No hay que olvidar que el $52 \%$ de los campos exige al socio registrarse para poder reservar en su página.

La trasparencia de la página Web se convierte en el principal portal para los campos de golf pues buscan mostrar al máximo todas sus virtudes y atraer al cliente. De este modo, la página Web se 
convierte en un elemento para cuantificar el rendimiento del campo sin necesidad de invertir en exceso en otros canales de promoción para atraer al mayor número de posible de clientes.

Además se podrían establecer una serie de líneas de investigación e implicaciones prácticas como:

- Establecer y analizar variables para cuantificar el rendimiento del campo mediante la página Web sin necesidad de invertir en exceso en otros canales de promoción, por lo que es recomendable que el campo de golf siga trabajando en la actualización y mantenimiento de las páginas Webs.

- Se debe estudiar el análisis sobre el diseño y disposición de la información más relevante para el usuarios donde se consigan los objetivos de a) convertirla en un elemento transparente y b) ser atractivo y accesible para que el usuario tenga la sensación de conocer el campo de primera mano desde la página Web.

- Es interesante analizar la satisfacción de los usuarios de golf sobre las páginas Web de los campos donde practican.

- Es necesario plantear un sistema de pago más fácil y rápido, para el socio y así poder reducir el gasto de tiempo al personal administrativo del club.

- Otras recomendaciones es la creación del ranking sobre el posicionamiento de los campos a nivel nacional e internacional, así como estudios económico-financieros si los clubes colgasen la información en sus páginas Web.

\section{Referencias}

ABC (2011). El turismo de golf supera los 680 millones de euros en Andalucía. Disponible online en: http://www.abcdesevilla.es (Fecha última consulta Julio, 2013).

Aguirre, P. (2013). Informe de transparencia del sector fundacional andaluz. Transparencia de las fundaciones en Internet. Sevilla: Asociación de Fundaciones Andaluzas.

Andrés, T., \& Gandía, J.L. (2000). Aportaciones de las nuevas tecnologías de la información a la mejora del modelo de comunicación empresarial. X Encuentro ASEPUC. 
Asociación Española de Contabilidad y Administración de Empresas. (1988). Documento $n^{\circ} 4$ de la Comisión de Nuevas Tecnologías y Contabilidad: Certificación y auditoria de la información digital. Madrid: Asociación Española de Contabilidad y Administración de Empresas.

Asociación Española de Contabilidad y Administración de Empresas. (2002). Documento $n^{o} 1$ de la Comisión de Nuevas Tecnologías y Contabilidad: Código de Buenas Prácticas para la divulgación de información financiera en Internet. Madrid: Asociación Española de Contabilidad y Administración de Empresas.

Asociación Española de Contabilidad y Administración de Empresas. (2004). Documento $n^{o} 3$ de la Comisión de Nuevas Tecnologías y Contabilidad: Información de empresas: análisis oferta-demanda y mecanismos de transmisión. Madrid: Asociación Española de Contabilidad y Administración de Empresas.

Aramayo-García, A., Arimany-Serrat, N., Uribe-Salazar, C., \& Sabata-Aliberch, A. (2016). Web communication of CSR and financial performance: Study applied to catalan meat companies. Intangible Capital, 12(2), 391-419. http://dx.doi.org/10.3926/ic.590

Barrio, E., \& Martín, J. (2012). Examen de transparencia. Informe de transparencia en la web de las Universidades Públicas Españolas. Madrid: Fundación Compromiso y Transparencia.

Barrio, E., Martín-Cavanna, J., \& Martisi, B.C. (2013). Transparencia, el mejor slogan 2012. Madrid: Fundación Compromiso Empresarial.

Berger, E., Brenne, T., Heath, A., Hochholdinger, B., Kassem-Manthey, K., Keßler, L., Koch, N., \& Wiegand, K. (2005). A benchmark study for different numerical parameters and their impact on the calculated strain levels for a model part door outer. AIP Conference Proceedings 778(A), 107-112. http://dx.doi.org/10.1063/1.2011202

Bonsón, E., \& Escobar, T. (2004). La difusión voluntaria de información financiera en Internet. Un análisis comparativo entre Estados Unidos, Europa del Este y la Unión Europea. Revista Española de Financiación y Contabilidad, 33, 1063-1101.

Bonsón, E., \& Escobar, T. (2006). Digital Reporting in Eastern Europe: An Empirical Study. International Journal of Accounting Information Systems, 7, 299-318.

http://dx.doi.org/10.1016/j.accinf.2006.09.001

Bonsón, E., Escobar, T., \& Gandía, J.L. (2004). Del código de buenas prácticas de AECA a la Ley de Transparencia. Revista de la Asociación Española de Contabilidady Administración de Empresas, 66, 46-50.

Bonsón, E., Escobar, T., \& Flores, F. (2006). Divulgación de información a través de Internet en el sector bancario. Comunicación presentada en jornadas de ASEPUC, Burgos. 2006. 
Borrero C.R., \& Cortijo, V. (2006). Transparencia de las páginas Web 2006 de las empresas del IBEX 35. Situación actual e implicaciones para el futuro. Boletin ICE Económico, 2896, 13-25.

Clark, J.M., Cornwell, T.B., \& Pruitt, S.W. (2009). The impact of title event sponsorship announcements on shareholder wealth. Marketing Letter, 20(2), 169-182. http://dx.doi.org/10.1007/s11002-008-9064-z

Codina, L. (2000a). Evaluación de recursos digitales en línea: conceptos, indicadores y métodos. Revista Española de Documentación Cientifica, 23(1), 9-44.

Codina, L. (2000b). Parámetros e indicadores de calidad para la evaluación de recursos digitales. En Jornadas Españolas de Documentación. La gestión del conocimiento: retos y soluciones de los profesionales de la información. Universidad del País Vasco, 135-144.

Confederación de Empresarios de Andalucía (CEA) (2000). Informe de turismo de golf. Consejería de Turismo y Comercio. Malaga: Junta de Andalucía.

Davis, CH.E., Clements, C., \& Kehuer, W.P. (2004). La información financiera a través de Internet. Harvard Deusto Finanzas y Contabilidad, 58, 69-73.

FASB (2000). Business Reporting Information Project, Electronic Distribution of Business Reporting Information. Disponible online en: http://www.fasb.org (Fecha última consulta: cotubre, 2014).

Gálvez, M.M., Caba, M.C., \& López, M. (2009). La transparencia on-line de las ONG españolas. Revista Española del Tercer Sector, 13, 63-86.

Gandía, J.L. (2001). La Divulgación de Información Financiera en la Era Digital. Comunicaciones de AECA. Madrid. Asociación Española de Contabilidad y Administración de Empresas.

Gandía, J.L. \& Andrés, T. (2005). E-Gobierno corporativo y transparencia informativa en las sociedades cotizadas españolas. Un estudio empirico de la Comisión Nacional del Mercado de Valores (CNMV). Monografía 8. Madrid: Comisión Nacional del Mercado de Valores.

Giner, B. (1997). The Influence of Company Characteristics and Accounting Regulation on Information Disclosed by Spanish Firms. The European Accounting Review, 6(1), 45-68. http://dx.doi.org/10.1080/096381897336863

Heald, D. (2006). Transparency as an instrumental value. En C. Hood \& D. Heald (Eds.). Transparency. The key to better governance?. Oxford: Oxford University Press for The British Academy.

Jadad, A., \& Gagliardi, A. (1998). Rating health information on the Internet. Navigating to knowledge or to Babel?. JAMA, 279, 611-614. http://dx.doi.org/10.1001/jama.279.8.611 
Larrán, M., \& Giner, B. (2002). The use of the Internet for corporate reporting by Spanish companies. The use of the Internet for corporate reporting by Spanish companies. The International Journal of Digital Accounting Research, 2, 53-82. http://dx.doi.org/10.4192/1577-8517-v2_3

Ley 10/1990, de 15 de octubre, del Deporte. Disponible online en: http://www.csd.gob.es/csd/informacion/legislacion-basica/ley-del-deporte (Fecha última consulta: octubre, 2014).

Ley 19/2013, de 9 de diciembre, de transparencia, acceso a la información pública y buen gobierno. Disponible online en: http://www.boe.es/boe/dias/2013/12/10/pdfs/BOE-A-2013-12887.pdf (Fecha última consulta: octubre, 2014).

Lymer, A., Debrency, R., Gray, G.L., \& Rahman, A. (1999). Business Reporting on the Internet. London: International Accounting standard.

Martí, M.L., Puertas, R., \& Calafat, C. (2014). Calidad y eficiencia de las Universidades Públicas Españolas. Revista de Estudios Regionales, 99, 134-154.

Meijer, A. (2009). Understanding modern transparency. International Review of Administrative Sciences, 75(2), 255-269. http://dx.doi.org/10.1177/0020852309104175

Merlo-Vega, J.A. (2003). La evaluación de la calidad de la información web: Aportaciones teóricas y experiencias prácticas. Recursos informativos: creación, descripción y evaluación. Sociedad de la información, 8, 101-110.

Muñoz, A., Del Campo, P., \& De La Fuente, D. (2001). Información financiera en Internet: Las sociedades del IBEX-35. Partida Doble, 119, 6-19.

Orden Eco/3722/2003, de 26 de diciembre, sobre el informe anual de Gobierno Corporativo y otros instrumentos de información de las sociedades anónimas cotizadas y otras entidades. BOE 8 de Enero de 2004. Disponible online en: https://www.cnmv.es/DocPortal/legislacion/ordenes/ECO 37222003. (Fecha última consulta: octubre, 2014).

Páez J.M., \& Oviedo, J.L. (2002). Divulgación de información financiera, social y medioambiental a través de Internet por las empresas españolas que cotizan en bolsa. XII Congreso AECA. (Asociación Española de Contabilidad y Administración de Empresas). Madrid: Empresa, Euro y Nueva Economía (pp. 26-28).

Proyecto De Ley De Transparencia Pública de Andalucía. Consejo de Gobierno, 18 de febrero de 2014. Junta de Andalucía. 
Rodríguez, F., \& Martín, J. (2013). Construir confianza 2013. Informe de transparencia y buen gobierno en la web de las fundaciones españolas. Madrid. Fundación Compromiso y Transparencia.

Rodríguez, M.P., \& Sáez J.L. (2005). Contabilidad y desarrollo de páginas web, como activo intangible generado internamente por la empresa. Técnica Contable, 676, 13-17.

Sanz, C.J., \& Bernal, J.A. (2008). Un análisis de la transparencia informativa por medio de las páginas web corporativas. Contaduría Universidad de Antioquia, 52, 85-104.

Sánchez, M. (1999). La Memoria como Cuenta Anual. Un estudio empirico. Tesis Doctoral. Universidad de Sevilla.

Sierra, G.J., \& Escobar, B. (1998). ¿Satisfacen las cuentas anuales las necesidades de información del usuario?. Partida Doble, 93, 12-17.

Sierra, G.J., \& Moreno, I. (2005). Valoración de la información financiera a través de web de las compañias del IBEX-35, Jornadas Hispano-Lusas de Gestión Científica.

Turespaña, Aymerich Golf Management y International Golf Travel Market (2005). Estudios sobre Turismo de golf. Madrid: Real Federación Española de Golf.

Intangible Capital, 2016 (www.intangiblecapital.org) 